Rapport - Société canadienne d'histoire de l'Église catholique

\title{
Un grand éducateur : Monseigneur François-Xavier Ross, premier évêque de Gaspé
}

\section{Michel Le Moignan}

Volume 32, 1965

URI : https://id.erudit.org/iderudit/1007332ar

DOI : https://doi.org/10.7202/1007332ar

Aller au sommaire du numéro

\section{Éditeur(s)}

Les Éditions Historia Ecclesiæ Catholicæ Canadensis Inc.

\section{ISSN}

0318-6148 (imprimé)

1927-7075 (numérique)

Découvrir la revue

\section{Citer cet article}

Le Moignan, M. (1965). Un grand éducateur : Monseigneur François-Xavier Ross, premier évêque de Gaspé. Rapport - Société canadienne d'histoire de l'Église catholique, 32, 67-73. https://doi.org/10.7202/1007332ar

Tous droits réservés @ Les Éditions Historia Ecclesiæ Catholicæ Canadensis Inc., 1966
Ce document est protégé par la loi sur le droit d'auteur. L'utilisation des services d'Érudit (y compris la reproduction) est assujettie à sa politique d'utilisation que vous pouvez consulter en ligne.

https://apropos.erudit.org/fr/usagers/politique-dutilisation/ 


\section{Un grand éducateur : Monseigneur François-Xavier Ross, premier évêque de Gaspé}

Au cours du siècle dernier, les évêques de Québec, face aux problèmes des régions lointaines mal pourvues de moyens de communication, avaient songé à l'organisation d'un nouveau diocèse dans la péninsule gaspésienne. Il en fut même question, bien avant l'érection du diocèse de Rimouski, en 1867.

En effet, dès 1849 , on étudiait déjà la possibilité d'ériger le district de Gaspé en un diocèse autonome. Comme document à l'appui, nous avons une lettre de Monseigneur Turgeon adressée à son grand vicaire, M. Mailloux, et datée du 25 janvier 1849 :

"Quant à la nomination d'un évêque pour le district de Gaspé, écrit-il, la difficulté de préposer un grand vicaire à cette partie du diocèse pourra l'accélérer, cependant je ne puis à mon grand regret regarder la chose comme très prochaine. Mais, en attendant qu'il en soit question, je vous prie de garder le plus strict tacet sur celui que vous m'indiquez comme pouvant être promu à l'épiscopat ${ }^{1}$. 》

Le projet n'eut pas de suite. Le premier diocèse qui fut créé par après fut celui de Rimouski, en 1867, incluant toute la Gaspésie.

Devenu évêque de Rimouski, en 1891, Mgr André-Albert Blais étudia de près la question. "Ce n'est pas un seul diocèse, mais deux qu'il faut en Gaspésie », écrivait-il, au début du siècle ${ }^{2}$. L'étude du projet se poursuivit pendant tout l'épiscopat de Mgr Blais, mais sans conclusion pratique.

La réalisation de ce dessein était réservée à feu Mgr Léonard, nommé évêque de Rimouski, en 1920. Né à Carleton, d'origine acadienne et gaspésienne, Mgr Léonard, dès le début de sa charge pastorale, chercha une solution au problème. Pour résumer, disons qu'une commission, présidée par son Vicaire général, Mgr François-Xavier Ross, suggéra de constituer le nouveau diocèse en détachant de Rimouski les comtés civils de Bonaventure et de Gaspé. Ainsi formé, le diocèse de Gaspé - trop vaste encore - devait s'étendre sur une distance de 350 milles, soit de Cap-Chat, dans Gaspé-Nord, jusqu'à Restigouche, dans la baie des Chaleurs. Sur cet immense territoire, on comptait seulement 37 paroisses, 14 missions et une mince population de 51,170 âmes, desservie par 55 prêtres.

1 Cité par C.-E. Roy, Gaspé depuis Cartier, 1934, p. 153.

2 Ibid., p. 153. 
Les membres de la commission, dans leur supplique adressée à Rome, fixaient le siège épiscopal à Gaspé, humble village perdu sur la côte-est, ne contenant qu'une population de 1,000 âmes, dont 600 catholiques. On admettait ces inconvénients immédiats. "Mais, précisaiton dans la requête, l'évêque est pour le diocèse et non le diocèse pour l'évêque. Et il n'est pas irrespectueux de présumer que ce seront là ses sentiments. Il sera là pour préparer l'avenir, non pour jouir du présent. Il s'agit de conserver ce pays et, au besoin, de le conquérir au prix de sacrifices. Comme le premier découvreur, le premier évêque plantera sa croix à Gaspé pour prendre possession du pays au nom du Christ ${ }^{3}$. ,

Ces paroles sont d'autant plus significatives que leur auteur deviendra, par la suite, le premier évêque de Gaspé. Quel est donc ce personnage?

Le 3 mai 1923, Monseigneur François-Xavier Ross n'arrivait pas en étranger dans sa ville épiscopale. Né aux portes de la Gaspésie, le 6 mars 1869, dans la mission des Grosses-Roches, le jeune Ross fit par la suite de brillantes études au petit Séminaire de Québec, au grand Séminaire de Rimouski, et plus tard à Rome. Le nouveau pasteur connaissait très bien son diocèse. Ex-secrétaire d'évêque, curé fondateur de quelques paroisses, principal d'Ecole normale et professeur de pédagogie, chanoine, vicaire général, protonotaire apostolique et vicaire capitulaire, Mgr Ross, âgé de 53 ans, s'attelait à une tâche immense, et pendant vingt-deux ans, "il mènera la bataille au pas de course ». L'un de ses collaborateurs intimes, le chanoine Camille LeBel, nous a tracé de lui le portrait suivant :

« Mgr Ross est alors, écrit-il, un petit homme sec et mince. Sa santé n'a jamais été très bonne. Nerveux, émotif, sensible à l'excès, il souffre de migraines, de névralgies, de lumbago. L'estomac travaille mal, les rhumes sont chez lui monnaie courante. Il dort peu et mal. Un défaut de langue le paralyse et l'empêche de donner sa mesure comme orateur. Devenu évêque, il ira suivre des traitements à Chicago et Rochester. On lui donnera des régimes et un rythme d'élocution qui répareront les plus gros dégâts de la nature. Mais dans cet organisme délâbré, l'énergie est superbe... En 1923, il arrive à Gaspé, avec ses complexes, sa fougue, son irritabilité, et... l'onction sainte de la plénitude du sacerdoce qui semble tout absorber ${ }^{4}$.

L'évêque prend donc possession de sa cathédrale, " une église pauvre, battue et mordue par le vent de la mer. ${ }^{5}$.» En ce moment, trois idées hantaient Mgr Ross, trois idées qu'il développera de façon magistrale dans son Mandement d'Entrée : en premier lieu, son idéal missionnaire; ensuite ses vues sur l'agriculture et la colonisation; enfin sa conception de l'éducation.

3 Ibid., p. 153.

4 Revue d'Histoire de la Gaspésie, janvier-mars 1964, p. 32.

5 Robert Ruminly, Histoire de la Province de Québec, t. XXVII, p. 13. 
Jeune prêtre, l'abbé Ross rêvait de devenir missionnaire. Plus tard, en 1921, il demandera même son admission au Séminaire des MissionsEtrangères récemment fondé. Cet idéal missionnaire, note dominante de sa vie, se concrétisera en 1928, alors que l'évêque fondera, à Gaspé, les Soeurs Missionnaires du Christ-Roi.

Comme nous n'avons pas l'intention d'analyser son rôle au chapître de l'agriculture et de la colonisation, retenons que Mgr Ross, comme la plupart des évêques de son temps, croyait encore à la vocation agricole du Québec.

Mais, allons tout de suite, dans l'un de ses champs d'apostolat où il a milité toute sa vie, celui de l'éducation. Dans son Mandement d'En. trée, l'évêque avouait « que l'une des principales raisons qui motivèrent la demande d'érection d'un diocèse en Gaspésie, fut la nécessité de pourvoir, par ce moyen, à l'organisation de maisons $d^{\prime}$ 'enseignement ${ }^{6}{ }^{8}$. Il est donc normal qu'il donne aux oeuvres d'éducation la primauté sur tout le reste. Mgr Ross est avant tout pasteur et éducateur. En effet, une longue expérience en ce domaine lui permettra de jouer un rôle de premier plan.

Sa carrière d'éducateur commence en 1906, au moment où il termine ses études en droit canonique à Rome. Encore dans la Ville Eternelle, il est nommé principal de l'Ecole normale de Rimouski que Mgr Blais vient de fonder. Tout reste à faire et il se met immédiatement au travail. C'est lui qui organise et dirige tout. Il sera l'âme de cette maison de formation pendant 17 ans, de 1906 à 1923. Pendant ces années de labeur acharné, le principal fait sa marque comme pédagogue. En 1910, et c'est lui-même qui l'écrit, « cette année et les années suivantes, le principal se charge d'enseigner lui-même, à tour de rôle, à peu près chacune de toutes les matières du programme, afin de constater par sa propre expérience ce qu'il est possible de donner à chaque matière, et de fixer le programme assimilable ${ }^{7}$.

Il faudrait relire les nombreuses conférences qu'il prononça devant les membres de la Société pédagogique et littéraire qu'il fonde à Rimouski, à la même époque, pour avoir une idée de sa conception de l'éducation. Nous y trouvons les grandes lignes de son programme futur. Nécessité de la formation des professeurs, amélioration du programme des écoles primaires, le besoin des études plus avancées pour les jeunes, etc. Telle conférence prononcée en 1913, par exemple, garde aujourd'hui encore toute son actualité. De nos jours, on place l'accent sur l'enfant. Mgr Ross ne pensait pas autrement. Ardent nationaliste, il voyait le salut de la race dans la parfaite coordination des efforts de la famille, de l'Eglise et de l'état. Permettez-moi de vous citer quelquesunes de ses paroles :

* L'Eglise et le pouvoir civil, affirmait-il, sont là pour guider les parents et les « aider * à remplir leur devoir d'éducation, ils ne doivent

6 Mandements des Evêques de Gaspé, t. 1, p. 24.

7 Ecole Normale de Rimouski, Annuaire, no 1, 1906 à 1917, p. 11. 
pas les supplanter, se substituer à eux. Je touche là une question des plus graves, la plus grave peut-être qui s'agite aujourd'hui dans le monde : je ne puis la traiter à fond, je me contente de vous donner les principes fondamentaux, si profondément ignorés par ceux qui barbouillent les journaux pour éclairer l'opinion ${ }^{8}$."

Le principal expose ensuite les droits respectifs des responsables de l'éducation des enfants, puis il ajoute ceci :

= Maie comme cette entente des narents pour le soutien des écoles, et le choix de maîtres compétent's, ne pourraient se faire sans le concours des autorités, l'Etat, disons le gouvernement, intervient, passe des lois qui assurent le bon fonctionnement des écoles, verse des allocations pour aider aux dépenses, et crée des foyers de formation pour les institutrices. C'est son devoir d'aider les individus à exercer leurs droits, rappelez-vous le bien. Parce que le gouvernement aide, ce n'est pas à conclure qu'il peut enlever aux parents leur droit d'éducation, leur imposer ses écoles, ses livres, ses instituteurs. L'école reste toujours le lieu où les parents exercent leur droit d'éducation. C'est sur le terrain de l'école que les adversaires de notre foi et de notre race nous livrent la lutte aujourd'hui. Celui qui est le maître de l'école est le maître des destinées d'un peuple, parce qu'il tient l'âme des enfants qui formeront la société de demain ${ }^{9}$.

Ces années fécondes l'amènent à publier, en 1913, son étude sur Louis Veuillot et la Presse catholique. En 1916, après une expérience de dix ans dans l'enseignement, il livre la première édition de son Manuel de pédagogie. Ouvrage qui fit sensation à l'époque et qui atteindra le $35^{\circ}$ mille à la mort de l'évêque. M. Gérard Filteau porte le jugement suivant sur ce manuel :

« Le nom de Mgr F.X. Ross est un de ceux qui s'imposent dans l'histoire de notre pédagogie. Principal auteur de la refonte du programme des écoles élémentaires, en 1923, il est' aussi l'auteur d'un Traité de pédagogie de haute valeur... La grande innovation de ce manuel est la large part faite à la psychologie de l'enfant. Sa méthodologie condense tous les progrès faits en ce domaine à l'époque ${ }^{10}$.

L'enseignement primaire fut l'une des préoccupations de la carrière de Mgr Ross. Sorti du peuple, il voyait le salut de la race canadiennefrançaise dans l'attachement' à la langue maternelle. C'est pour rester fidèle à cette conviction qu'il prit part, en 1920, à une violente polémique dans les journaux au sujet de l'introduction de la langue anglaise dans les écoles. C'est grâce à lui, si dans le temps, l'enseignement de cette langue ne commence pas avant la troisième année. On peut retrouver l'essentiel de ses arguments dans QUESTIONS SCOLAIRES, publié par le Devoir, en 1920.

8 Mgr Ross, Conférence sur l'Education, Rimouski, 1913.

9 Mgr Ross, Conférence sur l'Education, Rimouski, 1913.

10 Gérard Filteau, Organisation scolaire de la Province de Québec, p. 130-131. 
La préoccupation première de l'évêque de Gaspé, en 1923, avonsnous mentionné plus haut, le premier geste posé dès le lendemain de son intronisation, fut de s'attaquer au problème de l'éducation, certes le plus pressant de tous. Dans ce domaine, tout restait à bâtir. Pour les jeunes gens, aucune maison d'enseignement secondaire ou commercial. Pour les jeunes filles, quelques couvents peu développés. En fait d'enseignement technique, agricole ou autre, rien du tout. Organisation très déficiente à l'école primaire, aucune maison de formation pour les institutrices. Pour cet éducateur averti, il n'y a qu'un geste à poser : devenir à la fois fondateur et bâtisseur.

" L'histoire, affirmait un jour Mgr Ross, nous dit que Dieu ne fit dans son Eglise aucune des grandes oeuvres qui marquent une étape de l'humanité, sans y associer une femme ${ }^{11}$... D Dans le concret, l'évêque n'agira pas autrement. Un mois à peine, après son installation, il invite les Ursulines de Rimouski à fonder une Ecole normale à Gaspé.

Le 11 mai 1924, Mgr Ross assistait à la réunion du Conseil de l'Instruction publique où il fut question de la nomination d'un principal pour cette future école. "J'ai un candidat à vous proposer, dit Monseigneur : c'est un peu difficile pour moi de faire son éloge, mais j'ai confiance en lui... c'est l'évêque de Gaspé ${ }^{12}$. \ Evidemment, la suggestion fut adoptée à l'unanimité. Le 13 septembre, les 22 premières élèves s'installaient dans un local temporaire, et le principal continuera, pendant toute la durée de sa charge épiscopale, de faire bénéficier cette fondation de toute son expérience.

Le 7 octobre 1923, le pasteur du diocèse s'adressait à son clergé. "Désigné par la volonté du Seigneur, écrivait-il, pour gouverner ce diocèse où tout est à créer, sauf l'organisation paroissiale, nous avons porté nos regards vers le divin Maître pour chercher dans ses exemples la première ouvre que nous devons poser comme base de notre organisation ecclésiastique ${ }^{13}$. Vous l'avez deviné, sans nul doute, il s'agit de la fondation du petit Séminaire de Gaspé, confié aux Pères Jésuites, et qui ouvrira ses portes en septembre 1926. La même année, l'évêque invite les Hospitalières de Québec à fonder l'Hôtel-Dieu de Gaspé. Si on y regarde de près : Jésuites, Ursulines et Hospitalières, n'avons-nous pas là un trio historique dont $\mathbf{s}^{\prime}$ honore à juste titre le Canada français, et Mgr Ross se plaisait à dire : « Je recommence la fondation de la colonie.»

Poussant plus avant son désir d'éducation chrétienne et son idéal missionnaire, le pasteur fonda, en 1928, les Sœurs Missionnaires du Christ-Roi qui dispensent aujourd'hui l'instruction dans les missions indiennes de l'Ontario et de la Colombie Canadienne, sans oublier les écoles qu'elles dirigent au Congo et au Japon.

11 Conférence aux Ursulines de Gaspé, 1938.

12 Revue d'Histoire de la Gaspésie, juillet-septembre 1964, p. 157.

13 Mandements des Evêques de Gaspé, t. 1, p. 89. 
D'autres démarches aboutiront à l'organisation d'une Ecole moyenne d'agriculture, à Val d'Espoir, confiée aux Clercs de Saint-Viateur, et à la fondation d'une Ecole moyenne des Pêcheries, à Grande-Rivière. Certains projets, comme une Ecole de marine, une école de métiers, ne verront pas le jour immédiatement. Nous n'aurons pas d'école de marine, mais l'Ecole des Arts et Métiers de Gaspé viendra quand même après sa mort.

L'ambition première de l'évêque fut de donner à la jeunesse garpósienne toutes les possibilités dans le domaine de sa formation intellectuelle. Mais n'allons pas croire qu'il négligea, pour autant, l'éducation des adultes. Au lendemain de la première guerre mondiale, la Gaspésie tentait une première expérience dans le domaine de la coopération. Mais \& après que, faute de levain intérieur, les premières coopératives de pêcheurs eurent disparu, laissant les ouvriers de la mer désemparés, Mgr Ross n'abandonna pas la partie. Dix ans plus tard, alors que renaissait l'espérance d'une Gaspésie plus prospère, unie et maîtresse de ses affaires, sous le signe de la coopération, il était encore le premier sur la brèche ${ }^{14}$.

A son invitation, le Service Social-Economique de Sainte-Anne-dela-Pocatière incita les adultes à retourner sur les bancs de l'école. "Sans éducation, disait l'évêque, pas de coopération possible. " D'un bout à l'autre du diocèse, les cercles d'études s'organisent. De son bureau, Mgr Ross lance des pastorales lumineuses, devient professeur d'économie, car il faut renverser des positions solidement établies. Les fruits de cette éducation des adultes aboutirent à la mise sur pied d'un magnifique réseau de coopératives, couronné par la fondation des Pêcheurs-Unis du Québec. Dans la même ligne, c'est la multiplication des caisses populaires, des syndicats forestiers, des magasins coopératifs, des coopératives agricoles, etc.

Le grand éducateur ne cesse de donner des conférences, des cours de pédagogie. Il veut être partout à la fois, convaincu, dans le temps, que rien ne se fait en Gaspésie si le clergé ne prend les devants. Mais ici, comme ailleurs, obéissant à ses principes en éducation, l'Evêque demande au clergé de préparer des hommes pour occuper les postes, et non de vouloir tout diriger par lui-même.

L'une des armes les plus efficaces de Mgr Ross fut sa plume. Il écrivait avec une étonnante facilité. C'est une des raisons pour lesquelles la Société Royale du Canada l'admit dans ses rangs, en 1928. Il a laissé beaucoup de conférences, de sermons, trois gros volumes de Mandements et Lettres pastorales dont la plupart ont été reproduits par la Documentation Catholique de France.

Mgr François-Xavier Ross a laissé une empreinte durable en Gaspésie, non seulement comme éducateur, mais comme prêtre, apôtre et grand évêque. C'est lui qui a bâti le diocèse. 
* Gaspé est loin, déclarait-il un jour, sa position géographique en fait comme une excroissance dans laquelle la vie du corps social a circulé péniblement. On l'a oublié depuis Cartier qui l'a marquée de la croix, et le général Wolfe qui l'a marquée du fer rouge du conquérant. Cette péninsule a, pendant toute son histoire, porté cette double marque du Christ et du fer de l'étranger. Elle se réveille, et ranimant toutes les énergies latentes, elle rêve de reprendre, au soleil bienfaisant qui a fait épanouir la semence immortelle jetée jadis sur nos bords par la France chrétienne, la place que semblait lui prédestiner la première croix aux lys plantée en terre canadienne... Et pour réaliser ce rêve, elle a voulu commencer par fortifier ses positions en élevant comme des forteresses ces maisons d'éducation dans lesquelles se forme l'âme d'une race et se trempent les volontés d'un peuple qui doit prendre la direction de ses énergies ${ }^{15}$.

En juillet 1945, s'éteignait Mgr François-Xavier Ross, assistant au trône pontifical et comte romain depuis le 7 juillet 1934, chevalier de la Légion d'honneur, membre de l'Ordre du Mérite scolaire à titre de * Très Méritant \$.

Pour conclure, faisons nôtres ces paroles de M. Maurice LeBel, terminant sa notice biographique sur le premier évêque de Gaspé :

* Aux pieds du grand Christ blanc, dans le cimetière monastique des Ursulines où son corps repose, face à la baie qu'il aimait tant contempler et au milieu même des œuvres multiples que son dynamisme a su faire ériger, Mgr Ross peut envisager l'avenir avec confiance, car le souvenir de son passage et de son œuvre n'est pas près de s'éteindre, et son ouvre littéraire, mieux connue avec le temps, lui vaudra sans doute une place de choix dans les annales de la pédagogie, de la sociologie et de l'Eglise au Canada français. Il eût pu dire, lui aussi, comme Louis Veuillot, son maître :

Placez à mon côté ma plume;

Sur mon front le Christ, mon orgueil;

Sous mes pieds mettez ce volume,

Et clouez en paix ce cercueil ${ }^{16}$.

Michel Le Moignan, ptre

Président de la Société Historique de la Gaspésio

15 Discours de bienvenue lors de la visite du Surintendant de l'Instruction publique, à Gaspé, en 1928.

16 Maurice LEBEL, Le premier Evêque de Gaspé, CEuvre des Tracts, nº 381, p. 16. 